\title{
Inequalities for ranks of matrix expressions involving generalized inverses
}

\section{Zhiping Xiong*}

\section{"Correspondence:} xzpwhere@163.com

School of Mathematics and Computational Science, Wuy University, Jiangmen, 529020, P.R. China

\begin{abstract}
In this paper, we present several inequalities for ranks of the matrix expressions $D-A B X A B$ with respect to the choice of $X$, where $X$ is taken, respectively, as $B^{(1)} A^{(1)}$, $B^{(1,2)} A^{(1,2)}, B^{(1,3)} A^{(1,3)}, B^{(1,4)} A^{(1,4)}, B^{(1,2,3)} A^{(1,2,3)}$ as well as $B^{(1,2,4)} A^{(1,2,4)}$ and $B^{\dagger} A^{\dagger}$. Various application of these inequalities are also presented.

MSC: Primary 15A09; secondary 15A24

Keywords: matrix expression; generalized inverse; inequality; rank; generalized Schur complement
\end{abstract}

\section{Introduction}

Throughout this paper $C^{m \times n}$ denotes the set of all $m \times n$ matrices over the complex field $C$. $I_{k}$ denotes the identity matrix of order $k, O_{m \times n}$ is the $m \times n$ matrix of all zero entries (if no confusion occurs, we will drop the subscript). For a matrix $A \in C^{m \times n}, A^{*}, R(A), N(A)$ and $r(A)$ denote the conjugate transpose, the range space, the null space, and the rank of the matrix $A$, respectively.

For $A \in C^{m \times n}$, a generalized inverse $X$ of $A$ is a matrix which satisfies some of the following four Penrose equations [1]:
\{1 $\} \quad A X A=A$,
$\{2\} \quad X A X=X$,
$\{3\} \quad(A X)^{*}=A X$,
$\{4\} \quad(X A)^{*}=X A$.

For a subset $\{i, j, \ldots, k\}$ of the set $\{1,2,3,4\}$, the set of $n \times m$ matrices satisfying the equations $\{i\},\{j\}, \ldots,\{k\}$ from among equations $\{1\}-\{4\}$ is denoted by $A\{i, j, \ldots, k\}$. Arbitrary matrix from $A\{i, j, \ldots, k\}$ is called an $\{i, j, \ldots, k\}$-inverse of $A$ and is denoted by $A^{(i, j, \ldots, k)}$. For example, an $n \times m$ matrix $X$ of the set $A\{1\}$ is called a $g$-inverse of $A$ and is denoted by $X=A^{(1)}$. The well-known seven common types of generalized inverses of $A$ introduced from (1) are, respectively, the $\{1\}$-inverse ( $g$-inverse), $\{1,2\}$-inverse (reflexive $g$-inverse), $\{1,3\}$-inverse (least square $g$-inverse), $\{1,4\}$-inverse (minimum norm $g$-inverse), $\{1,2,3\}$ inverse, $\{1,2,4\}$-inverse and $\{1,2,3,4\}$-inverse. The unique $\{1,2,3,4\}$-inverse of $A$ is denoted by $A^{\dagger}$, which is called the Moore-Penrose inverse of $A$. For convenience, the symbols $E_{A}$ and $F_{A}$ stand for the two orthogonal projectors $E_{A}=I_{m}-A A^{\dagger}$ and $F_{A}=I_{n}-A^{\dagger} A$. We refer the reader to [2-4] for basic results on generalized inverses.

The notion of rank of a matrix appears to have been introduced [5], by Sylvester in 1851. Two principal classical results [6,7] on rank are Sylvester's law of nullity and Frobenius'

○2014 Xiong; licensee Springer. This is an Open Access article distributed under the terms of the Creative Commons Attribution License (http://creativecommons.org/licenses/by/2.0), which permits unrestricted use, distribution, and reproduction in any medium, provided the original work is properly cited. 
inequality. A modern inequality, obtained by Khatri [8] and Marsaglia [9], gives upper and lower bounds for the rank of the sum of two matrices.

Given a matrix expression with some variant matrices in it, the rank of the matrix expression will vary with respect to the variant matrices. Since the rank of matrix is an integer between 0 and the minimum of row and column numbers of the matrix [10], then the inequalities for ranks of matrix expressions must exist with respect to their variant matrices. Many problems in matrix theory and applications are closely related to the inequalities for ranks of matrix expressions with variant matrices. For example, a matrix expression $D-A X B$ of order $n$ is nonsingular if and only if the maximal rank of $D-A X B$ with respect to $X$ is $n$; a matrix equation $A X B=C$ is consistent if and only if the minimal rank of the matrix expression $C-A X B$ with respect to $X$ is zero; two consistent matrix equations $X_{1}=X_{1} A X_{1}$ and $X_{2}=X_{2} B X_{2}$ have a common solution if and only if the minimal rank of the difference $X_{1}-X_{2}$ of their solutions is zero. In general, for any two matrix expressions $P\left(X_{1}, X_{2}, \ldots, X_{m}\right)$ and $Q\left(Y_{1}, Y_{2}, \ldots, Y_{n}\right)$ of the same size, there are $X_{1}, X_{2}, \ldots, X_{m}$ and $Y_{1}, Y_{2}, \ldots, Y_{n}$ such that $P\left(X_{1}, X_{2}, \ldots, X_{m}\right)=Q\left(Y_{1}, Y_{2}, \ldots, Y_{n}\right)$ if and only if

$$
\min _{X_{1}, X_{2}, \ldots, X_{m} ; Y_{1}, Y_{2}, \ldots, Y_{n}} r\left(P\left(X_{1}, X_{2}, \ldots, X_{m}\right)-Q\left(Y_{1}, Y_{2}, \ldots, Y_{n}\right)\right)=0
$$

The inequalities for ranks of a matrix expression play the important roles in matrix theory for describing the dimension of the row and column vector space of the matrix expressions, which are well understand and are easy to compute by the well-known elementary or congruent matrix expressions, see, e.g., $[8,11-15]$. The inequalities for ranks of matrix expressions could be regarded as one of the fundamental topics in matrix theory and applications, which can be used to investigate nonsingularity and inverse of a matrix, range and rank invariance of a matrix, relations between subspaces, equalities of matrix expressions with variable matrices, reverse order laws for generalized inverses, existence of solutions to various matrix equations, and so on, see, e.g., [8, 9, 16-19].

In this paper, by using the maximal and minimal ranks of generalized Schur complement $[11,14]$, we get several inequalities for ranks of the matrix expressions $D-A B X A B$, where $X$ is taken, respectively, as $B^{(1)} A^{(1)}, B^{(1,2)} A^{(1,2)}, B^{(1,3)} A^{(1,3)}, B^{(1,4)} A^{(1,4)}, B^{(1,2,3)} A^{(1,2,3)}, B^{(1,2,4)} A^{(1,2,4)}$ and $B^{\dagger} A^{\dagger}$. We also derive various valuable consequences.

In order to find the inequalities for ranks of matrix expressions, we first mention the following lemmas, which will be used in this paper.

Lemma 1.1 [11,14] Let $A \in C^{m \times n}, B \in C^{m \times l}, C \in C^{k \times n}$ and $D \in C^{k \times l}$. Then

$$
\begin{aligned}
\max _{A^{(1)}} r\left(D-C A^{(1)} B\right)= & \min \left\{r(C, D), r\left(\begin{array}{l}
B \\
D
\end{array}\right), r\left(\begin{array}{ll}
A & B \\
C & D
\end{array}\right)-r(A)\right\}, \\
\min _{A^{(1)}} r\left(D-C A^{(1)} B\right)= & r(A)+r(C, D)+r\left(\begin{array}{l}
B \\
D
\end{array}\right)+r\left(\begin{array}{ll}
A & B \\
C & D
\end{array}\right) \\
& -r\left(\begin{array}{ll}
A & O \\
O & B \\
C & D
\end{array}\right)-r\left(\begin{array}{lll}
A & O & B \\
O & C & D
\end{array}\right), \\
\max _{A^{(1,2)}} r\left(D-C A^{(1,2)} B\right)= & \min \left\{r(A)+r(D), r(C, D), r\left(\begin{array}{l}
B \\
D
\end{array}\right), r\left(\begin{array}{ll}
A & B \\
C & D
\end{array}\right)-r(A)\right\},
\end{aligned}
$$




$$
\min _{A^{(1,2)}} r\left(D-C A^{(1,2)} B\right)=r\left(\begin{array}{l}
B \\
D
\end{array}\right)+r(C, D)+r(A)+\max \left\{S_{1}, S_{2}\right\}
$$

where

$$
\begin{aligned}
& S_{1}=r\left(\begin{array}{ll}
A & B \\
C & D
\end{array}\right)-r\left(\begin{array}{lll}
A & O & B \\
O & C & D
\end{array}\right)-r\left(\begin{array}{ll}
A & O \\
O & B \\
C & D
\end{array}\right), \\
& S_{2}=r(D)-r\left(\begin{array}{ll}
A & O \\
C & D
\end{array}\right)-r\left(\begin{array}{ll}
A & B \\
O & D
\end{array}\right), \\
& \left.\max _{A^{(1,3)}} r\left(D-C A^{(1,3)} B\right)=\min \left(\begin{array}{cc}
A^{*} A & A^{*} B \\
r & D
\end{array}\right)-r(A), r\left(\begin{array}{l}
B \\
D
\end{array}\right)\right\}, \\
& \min _{A^{(1,3)}} r\left(D-C A^{(1,3)} B\right)=r\left(\begin{array}{cc}
A^{*} A & A^{*} B \\
C & D
\end{array}\right)+r\left(\begin{array}{ll}
A & O \\
D
\end{array}\right)-r\left(\begin{array}{cc}
O & B \\
C & D
\end{array}\right), \\
& r\left(D-C A^{\dagger} B\right)=r\left(\begin{array}{cc}
A^{*} A A^{*} & A^{*} B \\
C A^{*} & D
\end{array}\right)-r(A) .
\end{aligned}
$$

Lemma 1.2 [20] Suppose $B, C$ and $D$ satisfy $R(D) \subseteq R(C)$ and $R\left(D^{*}\right) \subseteq R\left(B^{*}\right)$. Then the Moore-Penrose inverse of the block matrix

$$
M=\left(\begin{array}{ll}
O & B \\
C & D
\end{array}\right)
$$

can be expressed as

$$
M^{\dagger}=\left(\begin{array}{ll}
O & B \\
C & D
\end{array}\right)^{\dagger}=\left(\begin{array}{cc}
-C^{\dagger} D B^{\dagger} & C^{\dagger} \\
B^{\dagger} & O
\end{array}\right)
$$

Lemma 1.3 [19] Let $A \in C^{m \times n}, B \in C^{m \times k}, C \in C^{p \times n}$ and $D \in C^{p \times k}$. Then

(I) $r(A, B)=r(A)+r\left(E_{A} B\right)=r\left(E_{B} A\right)+r(B)$,

(II) $r(A, B)=r(A)+r(B)-\operatorname{dim}(R(A) \cap R(B))$,

(III) $r(A, B) \leq r(A)+r(B)$,

(IV) $r\left(\begin{array}{l}A \\ C\end{array}\right)=r(A)+r\left(C F_{A}\right)=r\left(A F_{C}\right)+r(C) \leq r(A)+r(C)$,

(V) $r\left({ }_{C}^{A}\right)=r(A)+r(C)-\operatorname{dim}(N(A) \cap N(C))$,

(VI) $r\left(\begin{array}{ll}A & B \\ C & D\end{array}\right)=r(A)+r\left(C F_{A}\right)+r\left(E_{A} B\right)+r\left(E_{C_{1}} S_{A} F_{B_{1}}\right)$,

where $C_{1}=C F_{A}, B_{1}=E_{A} B, S_{A}=D-C A^{\dagger} B$ and $\operatorname{dim}$ denotes dimension.

\section{Inequalities for ranks of $D-A B B^{(1)} A^{(1)} A B$}

In this section, we will present several inequalities for ranks of the matrix expression $D$ $A B B^{(1)} A^{(1)} A B$, with respect to two variant matrices $B^{(1)} \in B\{1\}$ and $A^{(1)} \in A\{1\}$, where $A \in$ $C^{m \times n}, B \in C^{n \times p}$ and $D \in C^{m \times p}$ are given matrices. 
Theorem 2.1 Let $A \in C^{m \times n}, B \in C^{n \times p}$ and $D \in C^{m \times p}$. Then for any $B^{(1)} \in B\{1\}$ and $A^{(1)} \in$ $A\{1\}$, the following inequalities hold:

$$
\begin{aligned}
r\left(D-A B B^{(1)} A^{(1)} A B\right) \leq & \min \left\{r(A B, D), r\left(\begin{array}{c}
A B \\
D
\end{array}\right),\right. \\
& n+r(A B)+r(D-A B)-r(A)-r(B)\}, \\
r\left(D-A B B^{(1)} A^{(1)} A B\right) \geq & r\left(\begin{array}{c}
A B \\
D
\end{array}\right)+r(D-A B)-r(A B) \\
& -\min \left\{r\left(\begin{array}{cc}
A B & D \\
O & A B
\end{array}\right)-r(A B), n+r\left(\begin{array}{c}
A B \\
D
\end{array}\right)-r(A)-r(B)\right\} .
\end{aligned}
$$

Proof Using formula (2) in Lemma 1.1 and formula (IV) in Lemma 1.3, we have

$$
\begin{aligned}
\max _{A^{(1)}} & r\left(D-A B B^{(1)} A^{(1)} A B\right) \\
& =\min \left\{r\left(A B B^{(1)}, D\right), r\left(\begin{array}{c}
A B \\
D
\end{array}\right), r\left(\begin{array}{cc}
A & A B \\
A B B^{(1)} & D
\end{array}\right)-r(A)\right\} \\
& =\min \left\{r\left(A B B^{(1)}, D\right), r\left(\begin{array}{c}
A B \\
D
\end{array}\right), r\left(A B B^{(1)} F_{A}, D-A B\right)\right\} \\
& =\min \left\{r\left(\begin{array}{c}
A B \\
D
\end{array}\right), r\left(A B B^{(1)} F_{A}, D-A B\right)\right\} .
\end{aligned}
$$

The last equation holds, since

$$
\begin{aligned}
r\left(A B B^{(1)} F_{A}, D-A B\right) & =r\left(\left(A B B^{(1)}, D-A B\right)\left(\begin{array}{cc}
F_{A} & O \\
O & I_{p}
\end{array}\right)\right) \\
& \leq r\left(A B B^{(1)}, D-A B\right) \\
& =r\left[\left(A B B^{(1)}, D-A B\right)\left(\begin{array}{cc}
I_{n} & B \\
O & I_{p}
\end{array}\right)\right] \\
& =r\left(A B B^{(1)}, D\right),
\end{aligned}
$$

i.e. $r\left(A B B^{(1)} F_{A}, D-A B\right) \leq r\left(A B B^{(1)}, D\right)$.

Using formula (2) in Lemma 1.1 again, we have

$$
\begin{aligned}
\max _{B^{(1)}} & r\left(A B B^{(1)} F_{A}, D-A B\right) \\
\quad & \max _{B^{(1)}} r\left([D-A B, O]+A B B^{(1)}\left[O, F_{A}\right]\right) \\
& =\min \left\{r(A B, D-A B), r\left(F_{A}\right)+r(D-A B), r\left(\begin{array}{ccc}
B & O & F_{A} \\
A B & D-A B & O
\end{array}\right)-r(B)\right\} \\
& =\min \left\{r(A B, D), r\left(F_{A}\right)+r(D-A B), r\left(B, F_{A}\right)+r(D-A B)-r(B)\right\}
\end{aligned}
$$




$$
\begin{aligned}
& =\min \left\{r(A B, D), r\left(B, F_{A}\right)+r(D-A B)-r(B)\right\} \\
& =\min \{r(A B, D), n+r(A B)+r(D-A B)-r(A)-r(B)\} .
\end{aligned}
$$

The third equation holds, since from formula (III) in Lemma 1.3,

$$
r\left(B, F_{A}\right) \leq r(B)+r\left(F_{A}\right)
$$

Combining (10) with (11), we have

$$
\begin{aligned}
& \max _{B^{(1)}, A^{(1)}} r\left(D-A B B^{(1)} A^{(1)} A B\right) \\
& \quad=\min \left\{r(A B, D), r\left(\begin{array}{c}
A B \\
D
\end{array}\right), n+r(A B)+r(D-A B)-r(A)-r(B)\right\} .
\end{aligned}
$$

That is, for any $B^{(1)} \in B\{1\}$ and $A^{(1)} \in A\{1\}$, the following inequalities hold:

$$
r\left(D-A B B^{(1)} A^{(1)} A B\right) \leq \min \left\{r(A B, D), r\left(\begin{array}{c}
A B \\
D
\end{array}\right), n+r(A B)+r(D-A B)-r(A)-r(B)\right\} .
$$

On the other hand, applying formula (3) in Lemma 1.1, we have

$$
\begin{aligned}
\min _{A^{(1)}} r\left(D-A B B^{(1)} A^{(1)} A B\right)= & r(A)+r\left(A B B^{(1)}, D\right)+r\left(\begin{array}{c}
A B \\
D
\end{array}\right)+r\left(\begin{array}{cc}
A & A B \\
A B B^{(1)} & D
\end{array}\right) \\
& -r\left(\begin{array}{ccc}
A & O & A B \\
O & A B B^{(1)} & D
\end{array}\right)-r\left(\begin{array}{cc}
A & O \\
O & A B \\
A B B^{(1)} & D
\end{array}\right) \\
= & r\left(\begin{array}{c}
A B \\
D
\end{array}\right)+r\left(\begin{array}{ccc}
A & A B \\
A B B^{(1)} & D
\end{array}\right)-r\left(\begin{array}{cc}
A & O \\
O & A B \\
A B B^{(1)} & D
\end{array}\right) .
\end{aligned}
$$

According to (13), we have

$$
\begin{aligned}
\min _{B^{(1)}, A^{(1)}} r\left(D-A B B^{(1)} A^{(1)} A B\right) \geq & r\left(\begin{array}{c}
A B \\
D
\end{array}\right)+\min _{B^{(1)}} r\left(\begin{array}{cc}
A & A B \\
A B B^{(1)} & D
\end{array}\right) \\
& -\max _{B^{(1)}} r\left(\begin{array}{cc}
A & O \\
O & A B \\
A B B^{(1)} & D
\end{array}\right) .
\end{aligned}
$$

By formula (3) in Lemma 1.1 and formula (IV) in Lemma 1.3, we have

$$
\begin{aligned}
\min _{B^{(1)}} r\left(\begin{array}{cc}
A & A B \\
A B B^{(1)} & D
\end{array}\right) & =r(A)+\min _{B^{(1)}} r\left(A B B^{(1)} F_{A}, D-A B\right) \\
& =r(A)+\min _{B^{(1)}} r\left([D-A B, O]+A B B^{(1)}\left[O, F_{A}\right]\right) \\
& =r(A)+r(D-A B) .
\end{aligned}
$$


Using formula (2) in Lemma 1.1 and formulas (III), (IV) in Lemma 1.3, we have

$$
\begin{aligned}
\max _{B^{(1)}} r\left(\begin{array}{cc}
A & O \\
O & A B \\
A B B^{(1)} & D
\end{array}\right) \\
=r(A)+r(A B)+\max _{B^{(1)}} r\left(A B B^{(1)} F_{A}, D F_{A B}\right) \\
=r(A)+r(A B)+\max _{B^{(1)}} r\left(\left[D F_{A B}, O\right]+A B B^{(1)}\left[O, F_{A}\right]\right) \\
=r(A)+r(A B)+\min _{2}\left\{r\left(A B, D F_{A B}\right), r\left(B, F_{A}\right)+r\left(D F_{A B}\right)-r(B)\right\} \\
=r(A)+r(A B)+\min \left\{r\left(\begin{array}{cc}
A B & D \\
O & A B
\end{array}\right)-r(A B), n+r\left(\begin{array}{c}
A B \\
D
\end{array}\right)-r(A)-r(B)\right\} .
\end{aligned}
$$

Combining the formulas (14), (15) with (16), we obtain

$$
\begin{aligned}
\min _{B^{(1)}, A^{(1)}} r\left(D-A B B^{(1)} A^{(1)} A B\right) & \\
\geq & r\left(\begin{array}{c}
A B \\
D
\end{array}\right)+r(D-A B)-r(A B) \\
& -\min \left\{r\left(\begin{array}{cc}
A B & D \\
O & A B
\end{array}\right)-r(A B), n+r\left(\begin{array}{c}
A B \\
D
\end{array}\right)-r(A)-r(B)\right\} .
\end{aligned}
$$

That is, for any $B^{(1)} \in B\{1\}$ and $A^{(1)} \in A\{1\}$, the following inequality holds:

$$
\begin{aligned}
r(D- & \left.A B B^{(1)} A^{(1)} A B\right) \\
\geq & r\left(\begin{array}{c}
A B \\
D
\end{array}\right)+r(D-A B)-r(A B) \\
& \quad-\min \left\{r\left(\begin{array}{cc}
A B & D \\
O & A B
\end{array}\right)-r(A B), n+r\left(\begin{array}{c}
A B \\
D
\end{array}\right)-r(A)-r(B)\right\} .
\end{aligned}
$$

Substituting in Theorem 2.1 with $D=A B$, we immediately obtain the following corollaries.

Corollary 2.1 ([21, Theorem 2.2], [22, Theorem 2.3]) For any matrices $A \in C^{m \times n}$ and $B \in C^{n \times p}$, the identity $A B=A B B^{(1)} A^{(1)} A B$ holds for any $B^{(1)} \in B\{1\}$ and $A^{(1)} \in A\{1\}$ if and only if

$$
A B=O \quad \text { or } \quad n+r(A B)=r(A)+r(B) .
$$

In particular, substituting in Theorem 2.1 with $D=O$ leads to the following result.

Corollary 2.2 Let $A \in C^{m \times n}$ and $B \in C^{n \times p}$. Then for any $B^{(1)} \in B\{1\}$ and $A^{(1)} \in A\{1\}$, the following inequalities holds:

$$
r(A B)-\min \{r(A B), r(A B)+n-r(A)-r(B)\} \leq r\left(A B B^{(1)} A^{(1)} A B\right) \leq r(A B) .
$$


Corollary 2.3 Let $A \in C^{m \times n}$ and $B \in C^{n \times p}$. Then the identity

$$
R\left(A B B^{(1)} A^{(1)} A B\right)=R(A B)
$$

holds, for any $B^{(1)} \in B\{1\}$ and $A^{(1)} \in A\{1\}$, if and only if

$$
A B=O \quad \text { or } \quad n+r(A B)=r(A)+r(B) .
$$

\section{Inequalities for ranks of $D-A B B^{(1,2)} A^{(1,2)} A B$}

By analogy with the proof of Theorem 2.1, in this section we will present several inequalities for ranks of the matrix expression $D-A B B^{(1,2)} A^{(1,2)} A B$. The main result in this section is the following theorem.

Theorem 3.1 Let $A \in C^{m \times n}, B \in C^{n \times p}$ and $D \in C^{m \times p}$. Then for any $B^{(1,2)} \in B\{1,2\}$ and $A^{(1,2)} \in A\{1,2\}$, the following inequalities hold:

$$
\begin{aligned}
r\left(D-A B B^{(1,2)} A^{(1,2)} A B\right) \leq & \min \left\{r\left(\begin{array}{c}
A B \\
D
\end{array}\right), r(A B, D),\right. \\
& n+r(A B)+r(D-A B)-r(A)-r(B)\}, \\
r\left(D-A B B^{(1,2)} A^{(1,2)} A B\right) \geq & r(A)+r\left(\begin{array}{c}
A B \\
D
\end{array}\right)+r(A B, D)+\max \left\{T_{1}, T_{2}\right\},
\end{aligned}
$$

where

$$
\begin{aligned}
T_{1}= & r(D-A B)-r(A B, D)-r(A B)-r(A) \\
& -\min \left\{r\left(\begin{array}{cc}
A B & D \\
O & A B
\end{array}\right)-r(A B), n+r\left(\begin{array}{c}
A B \\
D
\end{array}\right)-r(A)-r(B)\right\}, \\
T_{2}= & -2 r(A)-\min \{r(A B, D), n+r(A B)+r(D)-r(A)-r(B)\} .
\end{aligned}
$$

Proof Applying formula (4) in Lemma 1.1 and the formulas (III), (IV) in Lemma 1.3, we have

$$
\begin{aligned}
\max _{A^{(1,2)}} & r\left(D-A B B^{(1,2)} A^{(1,2)} A B\right) \\
& =\min \left\{r(A)+r(D), r\left(A B B^{(1,2)}, D\right), r\left(\begin{array}{c}
A B \\
D
\end{array}\right), r\left(\begin{array}{cc}
A & A B \\
A B B^{(1,2)} & D
\end{array}\right)-r(A)\right\} \\
& =\min \left\{r\left(\begin{array}{c}
A B \\
D
\end{array}\right), r\left(A B B^{(1,2)} F_{A}, D-A B\right)\right\} .
\end{aligned}
$$

The second equation holds, since $r\left(\begin{array}{c}A B \\ D\end{array}\right) \leq r(A B)+r(D) \leq r(A)+r(D)$, and

$$
\begin{aligned}
r\left(A B B^{(1,2)} F_{A}, D-A B\right) & =r\left(\left(A B B^{(1,2)}, D-A B\right)\left(\begin{array}{cc}
F_{A} & O \\
O & I_{p}
\end{array}\right)\right) \\
& \leq r\left(A B B^{(1,2)}, D-A B\right) .
\end{aligned}
$$


Applying the formulas (4) in Lemma 1.1 again, we have

$$
\begin{aligned}
& \max _{B^{(1,2)}} r\left(A B B^{(1,2)} F_{A}, D-A B\right) \\
& =\max _{B^{(1,2)}} r\left([D-A B, O]+A B B^{(1,2)}\left[O, F_{A}\right]\right) \\
& =\min \left\{r(A B, D-A B), r\left(F_{A}\right)+r(D-A B), r(B)+r(D-A B),\right. \\
& \left.r\left(\begin{array}{ccc}
B & O & F_{A} \\
A B & D-A B & O
\end{array}\right)-r(B)\right\} \\
& =\min \left\{r(A B, D), r\left(B, F_{A}\right)+r(D-A B)-r(B)\right\} \\
& =\min \{r(A B, D), n+r(A B)+r(D-A B)-r(A)-r(B)\} \text {. }
\end{aligned}
$$

The third equation holds, since from formula (III) in Lemma 1.3

$$
r\left(B, F_{A}\right) \leq r(B)+r\left(F_{A}\right)
$$

and

$$
r(A B, D)=r(A B, D-A B) \leq r(B)+r(D-A B) .
$$

In view of (18) and (19) it follows that

$$
\begin{aligned}
& \max _{B^{(1,2)}, A^{(1,2)}} r\left(D-A B B^{(1,2)} A^{(1,2)} A B\right) \\
& =\min \left\{r\left(\begin{array}{c}
A B \\
D
\end{array}\right), r(A B, D), n+r(A B)+r(D-A B)-r(A)-r(B)\right\} .
\end{aligned}
$$

That is, for any $B^{(1,2)} \in B\{1,2\}$ and $A^{(1,2)} \in A\{1,2\}$, the following inequalities hold:

$$
\begin{aligned}
r\left(D-A B B^{(1,2)} A^{(1,2)} A B\right) \leq & \min \left\{r(A B, D), r\left(\begin{array}{c}
A B \\
D
\end{array}\right),\right. \\
& n+r(A B)+r(D-A B)-r(A)-r(B)\} .
\end{aligned}
$$

On the other hand, using formula (5) in Lemma 1.1 and formula (I) in Lemma 1.3, we have

$$
\begin{aligned}
\min _{A^{(1,2)}} & r\left(D-A B B^{(1,2)} A^{(1,2)} A B\right) \\
& =r\left(\begin{array}{c}
A B \\
D
\end{array}\right)+r\left(A B B^{(1,2)}, D\right)+r(A)+\max \left\{\bar{S}_{1}, \bar{S}_{2}\right\} \\
& =r\left(\begin{array}{c}
A B \\
D
\end{array}\right)+r(A B, D)+r(A)+\max \left\{\bar{S}_{1}, \bar{S}_{2}\right\},
\end{aligned}
$$


where

$$
\begin{aligned}
& \overline{S_{1}}=r\left(\begin{array}{cc}
A & A B \\
A B B^{(1,2)} & D
\end{array}\right)-r\left(\begin{array}{ccc}
A & O & A B \\
O & A B B^{(1,2)} & D
\end{array}\right)-r\left(\begin{array}{cc}
A & O \\
O & A B \\
A B B^{(1,2)} & D
\end{array}\right), \\
& \overline{S_{2}}=r(D)-r\left(\begin{array}{cc}
A & O \\
A B B^{(1,2)} & D
\end{array}\right)-r\left(\begin{array}{cc}
A & A B \\
O & D
\end{array}\right),
\end{aligned}
$$

and

$$
\begin{aligned}
r(A B, D) & =r\left(\left(A B B^{(1,2)}, D\right)\left(\begin{array}{ll}
B & O \\
O & I_{p}
\end{array}\right)\right) \\
& \leq r\left(A B B^{(1,2)}, D\right)=r\left((A B, D)\left(\begin{array}{cc}
B^{(1,2)} & O \\
O & I_{p}
\end{array}\right)\right) \leq r(A B, D) .
\end{aligned}
$$

According to the results in (21), we have

$$
\begin{aligned}
\min _{B^{(1,2)}, A^{(1,2)}} r\left(D-A B B^{(1,2)} A^{(1,2)} A B\right) \geq & r\left(\begin{array}{c}
A B \\
D
\end{array}\right)+r(A B, D)+r(A) \\
& +\max \left\{\min \overline{S_{1}}, \min \overline{S_{2}}\right\} .
\end{aligned}
$$

Note that, for any $B^{(1,2)} \in B\{1,2\}$,

$$
\min \bar{S}_{1} \geq\left(\min _{B^{(1,2)}} r\left(\begin{array}{cc}
A & A B \\
A B B^{(1,2)} & D
\end{array}\right)\right)-r(A)-r(A B, D)-\max _{B^{(1,2)}} r\left(\begin{array}{cc}
A & O \\
O & A B \\
A B B^{(1,2)} & D
\end{array}\right)
$$

and

$$
\min \bar{S}_{2} \geq-r(A)-\max _{B^{(1,2)}} r\left(\begin{array}{cc}
A & O \\
A B B^{(1,2)} & D
\end{array}\right) .
$$

Applying formula (5) in Lemma 1.1 and formula (IV) in Lemma 1.3, we have

$$
\begin{aligned}
\min _{B^{(1,2)}} r\left(\begin{array}{cc}
A & A B \\
A B B^{(1,2)} & D
\end{array}\right) & =r(A)+\min _{B^{(1,2)}} r\left(A B B^{(1,2)} F_{A}, D-A B\right) \\
& =r(A)+\min _{B^{(1,2)}} r\left([D-A B, O]+A B B^{(1,2)}\left[O, F_{A}\right]\right) \\
& =r(A)+r(D-A B)
\end{aligned}
$$

and

$$
\begin{aligned}
& \max _{B^{(1,2)}} r\left(\begin{array}{cc}
A & O \\
O & A B \\
A B B^{(1,2)} & D
\end{array}\right) \\
& =r(A)+r(A B)+\max _{B^{(1,2)}} r\left(A B B^{(1,2)} F_{A}, D F_{A B}\right)
\end{aligned}
$$




$$
\begin{aligned}
= & r(A)+r(A B)+\max _{B^{(1,2)}} r\left(\left[D F_{A B}, O\right]+A B B^{(1,2)}\left[O, F_{A}\right]\right) \\
= & \min \left\{r\left(\begin{array}{cc}
A B & D \\
O & A B
\end{array}\right)-r(A B), n+r\left(\begin{array}{c}
A B \\
D
\end{array}\right)-r(A)-r(B)\right\} \\
& +r(A)+r(A B) .
\end{aligned}
$$

Combining (23), (25) with (26), we have

$$
\begin{aligned}
\min \bar{S}_{1} \geq & r(D-A B)-r(A B, D)-r(A B)-r(A) \\
& -\min \left\{r\left(\begin{array}{cc}
A B & D \\
O & A B
\end{array}\right)-r(A B), n+r\left(\begin{array}{c}
A B \\
D
\end{array}\right)-r(A)-r(B)\right\} .
\end{aligned}
$$

That is,

$$
\min \bar{S}_{1} \geq T_{1}
$$

According to formula (4) in Lemma 1.1 and the formulas (III), (IV) in Lemma 1.3, we have

$$
\begin{aligned}
\max _{B^{(1,2)}} r\left(\begin{array}{cc}
A & O \\
A B B^{(1,2)} & D
\end{array}\right) \\
\quad=r(A)+\max _{B^{(1,2)}} r\left(A B B^{(1,2)} F_{A}, D\right) \\
=r(A)+\max _{B^{(1,2)}} r\left([D, O]+A B B^{(1,2)}\left[O, F_{A}\right]\right) \\
=r(A)+\min \left\{r(A B, D), r\left(B, F_{A}\right)+r(D)-r(B)\right\} \\
=r(A)+\min \{r(A B, D), n+r(A B)+r(D)-r(A)-r(B)\} .
\end{aligned}
$$

By (24) and (28), we have

$$
\min \bar{S}_{2} \geq-2 r(A)-\min \{r(A B, D), n+r(A B)+r(D)-r(A)-r(B)\}
$$

That is,

$$
\min \bar{S}_{2} \geq T_{2}
$$

Finally on account of (22), (27), and (29), it is seen that

$$
\min _{B^{(1,2)}, A^{(1,2)}} r\left(D-A B B^{(1,2)} A^{(1,2)} A B\right) \geq r\left(\begin{array}{c}
A B \\
D
\end{array}\right)+r(A B, D)+r(A)+\max \left\{T_{1}, T_{2}\right\} .
$$

That is, for any $B^{(1,2)} \in B\{1,2\}$ and $A^{(1,2)} \in A\{1,2\}$, we have

$$
r\left(D-A B B^{(1,2)} A^{(1,2)} A B\right) \geq r(A)+r\left(\begin{array}{c}
A B \\
D
\end{array}\right)+r(A B, D)+\max \left\{T_{1}, T_{2}\right\}
$$


From Theorem 3.1, we immediately obtain the following corollaries by the formulas (20) and (30).

Corollary 3.1 Let $A \in C^{m \times n}$ and $B \in C^{n \times p}$. Then the identity

$$
A B=A B B^{(1,2)} A^{(1,2)} A B
$$

holds for any $B^{(1,2)} \in B\{1,2\}$ and $A^{(1,2)} \in A\{1,2\}$ if and only if

$$
A B=O \quad \text { or } \quad n+r(A B)=r(A)+r(B) .
$$

In particular, substituting in Theorem 3.1 with $D=O$ leads to the following.

Corollary 3.2 Let $A \in C^{m \times n}$ and $B \in C^{n \times p}$. Then for any $B^{(1,2)} \in B\{1,2\}$ and $A^{(1,2)} \in A\{1,2\}$, the following inequalities holds:

$$
r(A B)-\min \{r(A B), r(A B)+n-r(A)-r(B)\} \leq r\left(A B B^{(1,2)} A^{(1,2)} A B\right) \leq r(A B) .
$$

Corollary 3.3 Let $A \in C^{m \times n}$ and $B \in C^{n \times p}$. Then the identity

$$
R\left(A B B^{(1,2)} A^{(1,2)} A B\right)=R(A B)
$$

holds for any $B^{(1,2)} \in B\{1,2\}$ and $A^{(1,2)} \in A\{1,2\}$ if and only if

$$
A B=O \quad \text { or } \quad n+r(A B)=r(A)+r(B) .
$$

\section{Inequalities for ranks of $D-A B B^{(1,3)} A^{(1,3)} A B$ and $D-A B B^{(1,4)} A^{(1,4)} A B$}

Applying the formulas (6) and (7) in Lemma 1.1 to the matrix expressions $D-A B B^{(1,3)} \times$ $A^{(1,3)} A B$ and $D-A B B^{(1,4)} A^{(1,4)} A B$, we obtain some inequalities for ranks of this two matrix expressions. The main result in this section is the following theorem.

Theorem 4.1 Let $A \in C^{m \times n}, B \in C^{n \times p}$ and $D \in C^{m \times p}$. Then for any $B^{(1,3)} \in B\{1,3\}$ and $A^{(1,3)} \in A\{1,3\}$, the following inequalities hold:

$$
\begin{aligned}
& r\left(D-A B B^{(1,3)} A^{(1,3)} A B\right) \\
& \quad \leq \min \left\{r\left(\begin{array}{ccc}
B^{*} B & O & B^{*} \\
A B & D-A B & O \\
O & O & A
\end{array}\right)-r(A)-r(B), r\left(\begin{array}{c}
A B \\
D
\end{array}\right)\right\}, \\
& r\left(D-A B B^{(1,3)} A^{(1,3)} A B\right) \\
& \quad \geq r\left(\begin{array}{ccc}
B^{*} B & O & B^{*} \\
A B & D-A B & O \\
O & O & A
\end{array}\right)+r\left(\begin{array}{c}
A B \\
D
\end{array}\right)-r\left(\begin{array}{ccc}
A B & O & D \\
B^{*} B & B^{*} & O \\
O & A & O \\
O & O & A B
\end{array}\right) .
\end{aligned}
$$


Proof According to formula (6) in Lemma 1.1 and formula (IV) in Lemma 1.3, we have

$$
\begin{aligned}
\max _{A^{(1,3)}} r\left(D-A B B^{(1,3)} A^{(1,3)} A B\right) \\
\quad=\min \left\{r\left(\begin{array}{c}
A B \\
D
\end{array}\right), r\left(\begin{array}{cc}
A^{*} A & A^{*} A B \\
A B B^{(1,3)} & D
\end{array}\right)-r(A)\right\} \\
\quad=\min \left\{r\left(\begin{array}{c}
A B \\
D
\end{array}\right), r\left(A B B^{(1,3)} F_{A}, D-A B\right)\right\} .
\end{aligned}
$$

Applying formula (6) in Lemma 1.1 and formula (IV) in Lemma 1.3 again, we have

$$
\begin{aligned}
\max _{B^{(1,3)}} r\left(A B B^{(1,3)} F_{A}, D-A B\right) & \\
& =\max _{B^{(1,3)}} r\left([D-A B, O]+A B B^{(1,3)}\left[O, F_{A}\right]\right) \\
& =\min \left\{r\left(F_{A}\right)+r(D-A B), r\left(\begin{array}{ccc}
B^{*} B & B^{*} F_{A} & O \\
A B & O & D-A B
\end{array}\right)-r(B)\right\} \\
& =r\left(\begin{array}{ccc}
B^{*} B & O & B^{*} \\
A B & D-A B & O \\
O & O & A
\end{array}\right)-r(A)-r(B) .
\end{aligned}
$$

The third equation holds, since from formula (III) in Lemma 1.3,

$$
\begin{aligned}
r\left(\begin{array}{ccc}
B^{*} B & B^{*} F_{A} & O \\
A B & O & D-A B
\end{array}\right) & \leq r\left(\begin{array}{cc}
B^{*} B & B^{*} F_{A} \\
A B & O
\end{array}\right)+r(D-A B) \\
& \leq r(B)+r\left(B^{*} F_{A}\right)+r(D-A B) \\
& \leq r(B)+r\left(F_{A}\right)+r(D-A B) .
\end{aligned}
$$

Combining (31) with (32), we have

$$
\begin{aligned}
& \max _{B^{(1,3)}, A^{(1,3)}} r\left(D-A B B^{(1,3)} A^{(1,3)} A B\right) \\
& =\min \left\{r\left(\begin{array}{ccc}
B^{*} B & O & B^{*} \\
A B & D-A B & O \\
O & O & A
\end{array}\right)-r(A)-r(B), r\left(\begin{array}{c}
A B \\
D
\end{array}\right)\right\} .
\end{aligned}
$$

That is, for any $B^{(1,3)} \in B\{1,3\}$ and $A^{(1,3)} \in A\{1,3\}$, the following inequalities hold:

$$
\begin{aligned}
& r\left(D-A B B^{(1,3)} A^{(1,3)} A B\right) \\
& \quad \leq \min \left\{r\left(\begin{array}{ccc}
B^{*} B & O & B^{*} \\
A B & D-A B & O \\
O & O & A
\end{array}\right)-r(A)-r(B), r\left(\begin{array}{c}
A B \\
D
\end{array}\right)\right\} .
\end{aligned}
$$


On the other hand, from formula (7) in Lemma 1.1 and formula (IV) in Lemma 1.3, we have

$$
\begin{aligned}
& \min _{A^{(1,3)}} r\left(D-A B B^{(1,3)} A^{(1,3)} A B\right) \\
& \quad=r\left(\begin{array}{c}
A B \\
D
\end{array}\right)+r\left(\begin{array}{cc}
A^{*} A & A^{*} A B \\
A B B^{(1,3)} & D
\end{array}\right)-r\left(\begin{array}{cc}
A & O \\
O & A B \\
A B B^{(1,3)} & D
\end{array}\right) \\
& \quad=r\left(A B B^{(1,3)} F_{A}, D-A B\right)+r\left(\begin{array}{c}
A B \\
D
\end{array}\right)-r(A B)-r\left(A B B^{(1,3)} F_{A}, D F_{A B}\right) .
\end{aligned}
$$

From (34), we get

$$
\begin{aligned}
& \min _{B^{(1,3)}, A^{(1,3)}} r\left(D-A B B^{(1,3)} A^{(1,3)} A B\right) \\
& \geq \min _{B^{(1,3)}}\left\{r\left(A B B^{(1,3)} F_{A}, D-A B\right)\right\}+r\left(\begin{array}{c}
A B \\
D
\end{array}\right)-r(A B) \\
& \quad-\max _{B^{(1,3)}} r\left(A B B^{(1,3)} F_{A}, D F_{A B}\right) .
\end{aligned}
$$

Applying formula (7) in Lemma 1.1 and formula (IV) in Lemma 1.3 again, we have

$$
\begin{aligned}
\min _{B^{(1,3)}} r\left(A B B^{(1,3)} F_{A}, D-A B\right) & \\
= & \min _{B^{(1,3)}} r\left([D-A B, O]+A B B^{(1,3)}\left[O, F_{A}\right]\right) \\
& =r(D-A B)-r\left(\begin{array}{cc}
B & O \\
A B & D-A B
\end{array}\right)+r\left(\begin{array}{ccc}
B^{*} B & B^{*} F_{A} & O \\
A B & O & D-A B
\end{array}\right) \\
& =r\left(\begin{array}{ccc}
B^{*} B & O & B^{*} \\
A B & D-A B & O \\
O & O & A
\end{array}\right)-r(A)-r(B) .
\end{aligned}
$$

Applying formula (6) in Lemma 1.1 and the formulas (I), (III) in Lemma 1.3, we have

$$
\begin{aligned}
\max _{B^{(1,3)}} & r\left(A B B^{(1,3)} F_{A}, D F_{A B}\right) \\
& =\max _{B^{(1,3)}} r\left(\left[D F_{A B}, O\right]+A B B^{(1,3)}\left[O, F_{A}\right]\right) \\
& =\min \left\{r\left(\begin{array}{ccc}
B^{*} B & B^{*} F_{A} & O \\
A B & O & D F_{A B}
\end{array}\right)-r(B), r\left(F_{A}\right)+r\left(D F_{A B}\right)\right\} \\
& =r\left(\begin{array}{ccc}
B^{*} B & B^{*} F_{A} & O \\
A B & O & D F_{A B}
\end{array}\right)-r(B) \\
& =r\left(\begin{array}{ccc}
B^{*} B & B^{*} & O \\
A B & O & D \\
O & A & O \\
O & O & A B
\end{array}\right)-r(A B)-r(A)-r(B) .
\end{aligned}
$$


The third equation holds, since

$$
\begin{aligned}
r\left(\begin{array}{ccc}
B^{*} B & B^{*} F_{A} & O \\
A B & O & D F_{A B}
\end{array}\right) & \leq r\left(\begin{array}{cc}
B^{*} B & B^{*} F_{A} \\
A B & O
\end{array}\right)+r\left(D F_{A B}\right) \\
& \leq r(B)+r\left(B^{*} F_{A}\right)+r\left(D F_{A B}\right) \\
& \leq r(B)+r\left(F_{A}\right)+r\left(D F_{A B}\right) .
\end{aligned}
$$

By (35), (36), and (37), we have

$$
\begin{aligned}
& \min _{B^{(1,3)}, A^{(1,3)}} r\left(D-A B B^{(1,3)} A^{(1,3)} A B\right) \\
& \geq r\left(\begin{array}{ccc}
B^{*} B & O & B^{*} \\
A B & D-A B & O \\
O & O & A
\end{array}\right)+r\left(\begin{array}{c}
A B \\
D
\end{array}\right)-r\left(\begin{array}{ccc}
A B & O & D \\
B^{*} B & B^{*} & O \\
O & A & O \\
O & O & A B
\end{array}\right) .
\end{aligned}
$$

That is, for any $B^{(1,3)} \in B\{1,3\}$ and $A^{(1,3)} \in A\{1,3\}$, we have

$$
\begin{aligned}
& r\left(D-A B B^{(1,3)} A^{(1,3)} A B\right) \\
& \geq r\left(\begin{array}{ccc}
B^{*} B & O & B^{*} \\
A B & D-A B & O \\
O & O & A
\end{array}\right)+r\left(\begin{array}{c}
A B \\
D
\end{array}\right)-r\left(\begin{array}{ccc}
A B & O & D \\
B^{*} B & B^{*} & O \\
O & A & O \\
O & O & A B
\end{array}\right) .
\end{aligned}
$$

From Theorem 4.1, we immediately obtain the following corollaries by (33) and (38).

Corollary 4.1 Let $A \in C^{m \times n}$ and $B \in C^{n \times p}$. Then the identity

$$
A B=A B B^{(1,3)} A^{(1,3)} A B
$$

holds for any $B^{(1,3)} \in B\{1,3\}$ and $A^{(1,3)} \in A\{1,3\}$ if and only if

$$
r(A B)+r\left(\begin{array}{c}
B^{*} \\
A
\end{array}\right)=r(A)+r(B) .
$$

Corollary 4.2 Let $A \in C^{m \times n}$ and $B \in C^{n \times p}$. Then for any $B^{(1,3)} \in B\{1,3\}$ and $A^{(1,3)} \in A\{1,3\}$, the following inequalities hold:

$$
r(A)+r(B)-r\left(\begin{array}{c}
B^{*} \\
A
\end{array}\right) \leq r\left(A B B^{(1,3)} A^{(1,3)} A B\right) \leq r(A B) .
$$

Corollary 4.3 Let $A \in C^{m \times n}$ and $B \in C^{n \times p}$. Then the identity

$$
R\left(A B B^{(1,3)} A^{(1,3)} A B\right)=R(A B)
$$


holds for any $B^{(1,3)} \in B\{1,3\}$ and $A^{(1,3)} \in A\{1,3\}$ if and only if

$$
r(A)+r(B)-r\left(\begin{array}{c}
B^{*} \\
A
\end{array}\right)=r(A B)
$$

Notice that a matrix $X$ belongs to $A\{1,4\}$ if and only if $X^{*}$ belongs to $A^{*}\{1,3\}$. So from the results obtained in above of Section 4, we can get the inequalities for ranks of $D$ $A B B^{(1,4)} A^{(1,4)} A B$. We state the following theorems without proofs.

Theorem 4.2 Let $A \in C^{m \times n}, B \in C^{n \times p}$ and $D \in C^{m \times p}$. Then for any $B^{(1,4)} \in B\{1,4\}$ and $A^{(1,4)} \in A\{1,4\}$, the following inequalities hold:

$$
\begin{aligned}
& r\left(D-A B B^{(1,4)} A^{(1,4)} A B\right) \\
& \quad \leq \min \left\{r\left(\begin{array}{ccc}
A A^{*} & O & A B \\
A^{*} & B & O \\
O & O & D-A B
\end{array}\right)-r(A)-r(B), r(A B, D)\right\}, \\
& r\left(D-A B B^{(1,4)} A^{(1,4)} A B\right) \\
& \quad \geq r\left(\begin{array}{ccc}
A A^{*} & O & A B \\
A^{*} & B & O \\
O & O & D-A B
\end{array}\right)+r(A B, D)-r\left(\begin{array}{cccc}
A A^{*} & O & O & A B \\
A^{*} & B & O & O \\
O & O & A B & D
\end{array}\right) .
\end{aligned}
$$

Corollary 4.4 Let $A \in C^{m \times n}$ and $B \in C^{n \times p}$. Then the identity

$$
A B=A B B^{(1,4)} A^{(1,4)} A B
$$

holds for any $B^{(1,4)} \in B\{1,4\}$ and $A^{(1,4)} \in A\{1,4\}$ if and only if

$$
r(A B)+r\left(A^{*}, B\right)=r(A)+r(B) .
$$

Corollary 4.5 Let $A \in C^{m \times n}$ and $B \in C^{n \times p}$. Then for any $B^{(1,4)} \in B\{1,4\}$ and $A^{(1,4)} \in A\{1,4\}$, the following inequalities hold:

$$
r(A)+r(B)-r\left(A^{*}, B\right) \leq r\left(A B B^{(1,3)} A^{(1,3)} A B\right) \leq r(A B) .
$$

Corollary 4.6 Let $A \in C^{m \times n}$ and $B \in C^{n \times p}$. Then the identity

$$
R\left(A B B^{(1,4)} A^{(1,4)} A B\right)=R(A B)
$$

holds for any $B^{(1,4)} \in B\{1,4\}$ and $A^{(1,4)} \in A\{1,4\}$ if and only if

$$
r(A)+r(B)-r\left(A^{*}, B\right)=r(A B) .
$$

\section{Inequalities for ranks of $D-A B B^{(1,2,3)} A^{(1,2,3)} A B$ and $D-A B B^{(1,2,4)} A^{(1,2,4)} A B$}

From the results in [3], it is seen that a matrix $X$ belongs to $B\{1,2,3\}$ if and only if

$$
X=\left(B^{*} B\right)^{(1)} B^{*},
$$


where $\left(B^{*} B\right)^{(1)} \in\left(B^{*} B\right)\{1\}$ is arbitrary. Similarly, a matrix $X$ belongs to $B\{1,2,4\}$ if and only if

$$
X=B^{*}\left(B B^{*}\right)^{(1)},
$$

where $\left(B B^{*}\right)^{(1)} \in\left(B B^{*}\right)\{1\}$ is arbitrary. Thus

$$
D-A B B^{(1,2,3)} A^{(1,2,3)} A B=D-A B\left(B^{*} B\right)^{(1)} B^{*}\left(A^{*} A\right)^{(1)} A^{*} A B
$$

and

$$
D-A B B^{(1,2,4)} A^{(1,2,4)} A B=D-A B B^{*}\left(B B^{*}\right)^{(1)} A^{*}\left(A A^{*}\right)^{(1)} A B .
$$

Applying the formulas (2) and (3) in Lemma 1.1 to (39) and (40), we have the following theorems, which can be shown by a similar approach to Theorem 2.1, and the proof are omitted here.

Theorem 5.1 Let $A \in C^{m \times n}, B \in C^{n \times p}$ and $D \in C^{m \times p}$. Then for any $B^{(1,2,3)} \in B\{1,2,3\}$ and $A^{(1,2,3)} \in A\{1,2,3\}$, the following inequalities hold:

$$
\begin{aligned}
& r\left(D-A B B^{(1,2,3)} A^{(1,2,3)} A B\right) \\
& \quad \leq \min \left\{r\left(\begin{array}{ccc}
B^{*} B & O & B^{*} \\
A B & D-A B & O \\
O & O & A
\end{array}\right)-r(A)-r(B), r\left(\begin{array}{c}
A B \\
D
\end{array}\right)\right\}, \\
& r\left(D-A B B^{(1,2,3)} A^{(1,2,3)} A B\right) \\
& \quad \geq r\left(\begin{array}{ccc}
B^{*} B & O & B^{*} \\
A B & D-A B & O \\
O & O & A
\end{array}\right)+r\left(\begin{array}{c}
A B \\
D
\end{array}\right)-r\left(\begin{array}{ccc}
A B & O & D \\
B^{*} B & B^{*} & O \\
O & A & O \\
O & O & A B
\end{array}\right) .
\end{aligned}
$$

Theorem 5.2 Let $A \in C^{m \times n}, B \in C^{n \times p}$ and $D \in C^{m \times p}$. Then for any $B^{(1,2,4)} \in B\{1,2,4\}$ and $A^{(1,2,4)} \in A\{1,2,4\}$, the following inequalities hold:

$$
\begin{aligned}
& r\left(D-A B B^{(1,2,4)} A^{(1,2,4)} A B\right) \\
& \quad \leq \min \left\{r\left(\begin{array}{ccc}
A A^{*} & O & A B \\
A^{*} & B & O \\
O & O & D-A B
\end{array}\right)-r(A)-r(B), r(A B, D)\right\}, \\
& r\left(D-A B B^{(1,2,4)} A^{(1,2,4)} A B\right) \\
& \quad \geq r\left(\begin{array}{ccc}
A A^{*} & O & A B \\
A^{*} & B & O \\
O & O & D-A B
\end{array}\right)+r(A B, D)-r\left(\begin{array}{cccc}
A A^{*} & O & O & A B \\
A^{*} & B & O & O \\
O & O & A B & D
\end{array}\right) .
\end{aligned}
$$




\section{Rank of $D-A B B^{\dagger} A^{\dagger} A B$}

In this section, we will present the rank of the linear matrix expression

$$
D-A B B^{\dagger} A^{\dagger} A B
$$

where $A \in C^{m \times n}, B \in C^{n \times p}$ and $D \in C^{m \times p}$ are given matrices.

Theorem 6.1 Let $A \in C^{m \times n}, B \in C^{n \times p}$ and $D \in C^{m \times p}$. Then

$$
r\left(D-A B B^{\dagger} A^{\dagger} A B\right)=r\left(\begin{array}{ccc}
O & A A^{*} & A B \\
B^{*} B & B^{*} A^{*} & O \\
A B & O & D
\end{array}\right)-r(A)-r(B) .
$$

Proof Let

$$
T=\left(\begin{array}{cc}
O & A A^{*} \\
B^{*} B & B^{*} A^{*}
\end{array}\right) .
$$

Then applying formula (9) in Lemma 1.2, we have

$$
\begin{aligned}
& T^{\dagger}=\left(\begin{array}{cc}
-B^{\dagger} A^{\dagger} & \left(B^{*} B\right)^{\dagger} \\
\left(A A^{*}\right)^{\dagger} & O
\end{array}\right), \quad T T^{\dagger}=\left(\begin{array}{cc}
A A^{\dagger} & O \\
O & B^{\dagger} B
\end{array}\right), \\
& T^{\dagger} T=\left(\begin{array}{cc}
B^{\dagger} B & O \\
O & A A^{\dagger}
\end{array}\right) .
\end{aligned}
$$

The sub-matrix in the upper left corner of the Moore-Penrose inverse of $T$ can be expressed as

$$
B^{\dagger} A^{\dagger}=-E_{1} T^{\dagger} E_{2}
$$

where $E_{1}=\left(I_{p}, O\right)$ and $E_{2}=\left(I_{m}, O\right)^{*}$. Hence

$$
D-A B B^{\dagger} A^{\dagger} A B=D+A B E_{1} T^{\dagger} E_{2} A B .
$$

Applying formula (8) in Lemma 1.1, we have

$$
\begin{aligned}
r\left(D-A B B^{\dagger} A^{\dagger} A B\right) & =r\left(D+A B E_{1} T^{\dagger} E_{2} A B\right) \\
& =r\left(\begin{array}{cc}
T^{*} T T^{*} & T^{*} E_{2} A B \\
-A B E_{1} T^{*} & D
\end{array}\right)-r(T) \\
& =r\left(\begin{array}{cc}
T & E_{2} A B \\
A B E_{1} & D
\end{array}\right)-r(T) \\
& =r\left(\begin{array}{ccc}
O & A A^{*} & A B \\
B^{*} B & B^{*} A^{*} & O \\
A B & O & D
\end{array}\right)-r(A)-r(B) .
\end{aligned}
$$

As a direct consequence of Theorem 6.1, we immediately get the following results. 
Corollary 6.1 Let $A \in C^{m \times n}$ and $B \in C^{n \times p}$. Then the identity $A B=A B B^{\dagger} A^{\dagger} A B$ holds if and only if

$$
r\left(\begin{array}{cc}
-A B & A A^{*} \\
B^{*} B & B^{*} A^{*}
\end{array}\right)+r(A B)=r(A)+r(B) .
$$

Corollary 6.2 Let $A \in C^{m \times n}$ and $B \in C^{n \times p}$. Then the identity $R\left(A B B^{\dagger} A^{\dagger} A B\right)=R(A B)$ holds if and only if

$$
r\left(\begin{array}{ccc}
O & A A^{*} & A B \\
B^{*} B & B^{*} A^{*} & O \\
A B & O & O
\end{array}\right)=r(A B)+r(A)+r(B) .
$$

\section{Competing interests}

The author declares that they have no competing interests.

\section{Acknowledgements}

The author would like to thank the Editor-in-Chief and the anonymous referees for their very detailed comments, which greatly improved the presentation of this article. The work was supported by the NSFC (Grant No: 11301397) and the Foundation for Distinguished Young Talents in Higher Education of Guangdong, China (2012LYM-0126) and the Basic Theory and Scientific Research of Science and Technology Project of Jiangmen City, China, 2013.

\section{Received: 18 September 2013 Accepted: 10 February 2014 Published: 20 Feb 2014}

\section{References}

1. Penrose, R: A generalized inverse for matrices. Proc. Camb. Philos. Soc. 51, 406-413 (1955)

2. Ben-Israel, A, Greville, TNE: Generalized Inverse: Theory and Applications. Wiley-Interscience, New York (1974); 2nd edn. Springer, New York (2002)

3. Rao, CR, Mitra, SK: Generalized Inverse of Matrices and Its Applications. Wiley, New York 1971

4. Wang, G, Wei, Y, Qiao, S: Generalized Inverses: Theory and Computations. Science Press, Beijing (2004)

5. Mirsky, L: An Introduction to Linear Algebra. Oxford University Press, Oxford (1955)

6. Frobenius, G: Über den rang einer matrix, vol. III, pp. 479-490. Springer, Berlin (1968)

7. Sylvester, JJ: The Collected Mathematical Papers of James Joseph Sylvester, vol. IV, pp. 133-145. Cambridge University Press, Cambridge (1912)

8. Khatri, CG: A simplified approach to the derivation of the theorems on the rank of a matrix. J. Maharaja Sayajirao Univ. Baroda 10, 1-5 (1961)

9. Marsaglia, G: Bounds on the rank of the sum of matrices. In: Trans. Fourth Prague Conf. on Information Theory, Statistical Decision Functions, Random Processes (Prague, August 31-September 11, 1965), pp. 455-462. Academia, Prague (1967)

10. David, CL: Linear Algebra and Its Applications. Addison-Wesley, Reading (1994)

11. Tian, Y: Upper and lower bounds for ranks of matrix expressions using generalized inverses. Linear Algebra Appl. 355 , 187-214 (2002)

12. Tian, Y: Ranks of solutions of the matrix equation $A X B=$ C. Linear Multilinear Algebra 51, 111-125 (2003)

13. Tian, $Y$, Cheng, S: The maximal and minimal ranks of $A-B X C$ with applications. N.Y. J. Math. 9, 345-362 (2003)

14. Tian, Y: More on maximal and minimal ranks of Schur complements with applications. Appl. Math. Comput. 152, 675-692 (2004)

15. Tian, Y: The maximal and minimal ranks of a quadratic matrix expression with applications. Linear Multilinear Algebra 59, 627-644 (2011)

16. Groß, J: Comment on range invariance of matrix products. Linear Multilinear Algebra 41, 157-160 (1996)

17. Groß, J, Tian, Y: Invariance properties of a triple matrix product involving generalized inverses. Linear Algebra Appl. 417, 94-107 (2006)

18. Johnson, CR, Whitney, GT: Minimum rank completions. Linear Multilinear Algebra 28, 271-273 (1991)

19. Marsaglia, G, Styan, GPH: Equalities and inequalities for ranks of matrices. Linear Multilinear Algebra 2, 269-292 (1974)

20. Hartwig, RE: Block generalized inverses. Arch. Ration. Mech. Anal. 61, 197-251 (1976)

21. Wei, M: Equivalent conditions for generalized inverses of products. Linear Algebra Appl. 266, 347-363 (1997)

22. Werner, $\mathrm{HJ}$ : When is $B^{-} A^{-}$a generalized inverse of $A B$ ? Linear Algebra Appl. 210, 255-263 (1994)

10.1186/1029-242X-2014-87

Cite this article as: Xiong: Inequalities for ranks of matrix expressions involving generalized inverses. Journal of Inequalities and Applications 2014, 2014:87 